\title{
ESTRATEGIAS DE AFRONTAMIENTO EN ADOLESCENTES
}

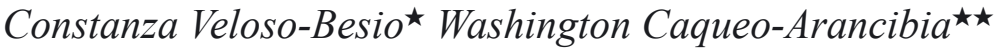 \\ Alejandra Caqueo-Urizar ${ }^{\star \star \star}$ Zunilda Muñoz-Sánchez ${ }^{\star \star \star \star}$ \\ Francisca Villegas-Abarzúa $a^{\star \star \star \star}$
}

\begin{abstract}
RESUMEN
Este estudio tuvo como objetivo describir y analizar las estrategias de afrontamiento que presentan los adolescentes escolarizados. La muestra total estuvo conformada por 323 adolecentes, estudiantes (184 hombres y 139 mujeres) de Octavo Básico a Cuarto Año Medio pertenecientes a diferentes tipos Establecimientos Educacionales de la ciudad de Arica (Chile): Privado (90), Particular Subvencionado (114) y Municipal (119). A todos ellos se les aplicó la escala ASC de Frydenberg y Lewis (1997). Se encontraron diferencias significativas entre los tipos de Establecimiento Educacional, siendo el Establecimiento Particular Subvencionado el que evidenció mayor variedad de estrategias de afrontamiento utilizadas. Se discuten las implicancias a la luz de los resultados hallados.
\end{abstract}

Palabras claves: estrategias de afrontamiento; adolescência; tipo de Establecimiento Educacional.

\section{Coping STRATEGIES IN ADOLESCENTS}

\begin{abstract}
This study aimed to describe and analyze the coping strategies that adolescents have in school. The total sample consisted of 323 adolescents, students (184 men and 139 women) from Eighth to Fourth Year Basic Middle belonging to different Educational Institutions in the city of Arica (Chile): Private (90), Private Subsidized (114) and Municipal (119). All of them received the ASC scale Frydenberg and Lewis (1997). There were significant differences between the types of Educational Establishments, with the individual establishment funded which showed greater variety of coping strategies used. Implications are discussed in light of the results found.
\end{abstract}

Keywords: coping strategies, adolescent, Educational Establishment type.

${ }^{\star}$ Departamento de Psicologia - Universidad de Tarapacá. 18 de Septiembre, 2222, Arica Chile.E-mail: veloso.constanza@gmail.com

$\star \star$ Académico Universidad de Tarapacá.E-mail: wcaqueo@uta.cl

$\star \star \star$ Académica Universidad de Tarapacá. E-mail: acaqueo@uta.cl

$\star \star \star \star$ Psicóloga Universidad de Tarapacá. E-mail: zunilda.msanchez@gmail.com

$\star \star \star \star \star$ Psicóloga Universidad de Tarapacá. E-mail: mfranciscavillegas@gmail.com 
Constanza Veloso-Besio; Washington Caqueo-Arancibia; Alejandra Caqueo-Urizar;

Zunilda Muñoz-Sánchez; Francisca Villegas-Abarzúa

\section{Estrategias DE AFRONTAMIENTO EN ADOLESCENTES EN DIFERENTES TIPOS DE ESTABLECIMIENTOS EDUCACIONALES}

\section{INTRODUCCIÓN: ADOLESCENCIA Y ESTRATEGIAS DE AFRONTAMIENTO}

La adolescencia es una etapa en que se experimentan cambios significativos en la personalidad; definición de identidad; elaboración de un proyecto de vida (FIGUEROA et al., 2005); dificultades en la toma de decisiones en relación con los estudios, y relaciones de pareja o actividad laboral (BALCÁZAR et al., 2006). Estos cambios pueden generar estrés en el adolescente (ISON, 2003), por lo que éste se ve en la necesidad de desarrollar competencias psicosociales importantes para su posterior posicionamiento en el mundo adulto. De no contar con estrategias de afrontamiento positivas para responder a este tipo de situaciones, se pueden generar comportamientos menos adaptativos y mayor vulnerabilidad en el adolescente, afectando su desarrollo psicológico (GONZÁLEZ et al., 2002) e incluso su vida (CUADRA et al., 2009).

De acuerdo con lo anterior, el psicólogo necesita conocer los modos de afrontamiento del adolescente, ya que tal información es útil para poder interpretar sus sentimientos, ideas y conductas (FRYDENBERG; LEWIS, 1997).

El constructo afrontamiento ha sido ampliamente estudiado, encontrándose en la literatura científica diferentes propuestas explicativas y clasificatorias (WILLIAMS; McGILLICUDDY, 2000), además de variados métodos de medición (ZIMER-GEMBECK; LOCKE, 2007). Dentro de este cuerpo, el modelo transaccional de Lazarus y Folkman (1984) es uno de los que más aceptación ha tenido entre los científicos. Aspecto que se ve claramente reflejado en la vasta cantidad de investigaciones que examinan los modos en que las personas enfrentan las dificultades y situaciones estresantes (AMIRKHAN; AUYEUNG, 2007; FEAGANS; HUSSONG; KEEKEY, 2008; FRYDENBERG et al., 2004; HAMPEL, 2007; HAMPEL; PETERMAN, 2006; PRITCHARD; WILSON, 2006).

Desde el modelo de Lazarus y Folkman (1984) no son las situaciones en sí las que provocan una reacción emocional, sino la interpretación que realiza el sujeto de tales emociones. Desde esta perspectiva, afrontamiento es entendido como:

[...] aquellos esfuerzos cognitivos y conductuales constantemente cambiantes que se desarrollan para manejar las demandas específicas externas y/o internas que son evaluadas como excedentes o desbordantes de los recursos del individuo (LAZARUS; FOLKMAN, 1984, p. 141).

Tales estrategias se van desarrollando por medio de las experiencias vividas durante la primera etapa de la vida (niñez) y se modifican de acuerdo a los resultados obtenidos en relación a las situaciones estresantes.

Lazarus y Folkman (1984) plantean que existen al menos dos amplios estilos diferentes de afrontamiento: "afrontamiento focalizado en el problema", que hace referencia a los esfuerzos dirigidos a modificar las solicitudes o eventos 
ambientales causantes del estrés, intentando solucionar el problema o al menos disminuir su impacto; y el "afrontamiento focalizado en las emociones", que implica realizar esfuerzos para aminorar o eliminar los sentimientos negativos causados por las condiciones estresantes. Mientras que Frydenberg e Lewis (1997) incorporan una tercera categoría a tales estilos, denominada "afrontamiento de evitación", que involucra evadir el problema, no prestando atención a él o distrayéndose con otras actividades o conductas.

Los dos primeros tipos de afrontamiento son fácilmente distinguibles y se pueden poner en práctica de forma paralela (CHICO, 2002). En cambio, el estilo centrado en la evitación es utilizado especialmente cuando las personas no quieren abocarse al problema, utilizando la evitación cognitiva (PINHEIRO; TROCCOLI; TAMAYO, 2003).

Los autores Frydenberg e Lewis (1997) afirman que es posible hacer una distinción entre afrontamiento general y específico, es decir el modo con el que el adolescente encara cualquier situación (general) y una problemática particular (específico). Es por esta razón que, los autores subdividen los tres estilos de afrontamiento en 18 estrategias diferentes: 1) estilo de afrontamiento focalizado en las emociones: buscar apoyo social, buscar pertenencia, invertir en amigos íntimos, acción social, buscar apoyo espiritual, buscar ayuda profesional; 2) estilo focalizado en la solución del problema: concentrarse en resolver el problema, esforzarse para tener éxito, fijarse en lo positivo, buscar diversiones relajantes, distracción física; 3) estilo focalizado en la evitación: preocuparse, hacerse ilusiones, falta de afrontamiento, reducción de la tensión, ignorar el problema, autoinculparse, reservarlo para sí.

En relación a la utilización de las estrategias, cada sujeto tiende a utilizar las estrategias de afrontamiento que domina, ya sea por aprendizaje previo o por hallazgo fortuito (CORRECHE; FANTIN; FLORENTINO, 2005). Estas estrategias estarían fuertemente condicionadas por la percepción que se haga de la situación (evaluación primaria) y de los recursos personales con que se cuenta para hacerle frente (evaluación secundaria) (GÓMEZ et al., 2006). A su vez, las estrategias de afrontamiento pueden resultar adaptativas, cuando reducen el estrés y promueven la salud a largo plazo, o inadaptativas cuando reducen el estrés sólo a corto plazo, provocando un efecto nocivo en la salud a largo plazo (FORNS; ZANINI, 2004). La adaptabilidad o inadaptabilidad de las estrategias de afrontamiento depende de la frecuencia con la que se utilicen (CORRECHÉ; FANTIN; FLORENTINO, 2005).

Es por esto que se ha generado un creciente interés por el estudio de las estrategias de afrontamiento, producto de reconocer en ellas un particular modo de responder a las diferentes situaciones que generan estrés, como también de entender su función positiva de mitigar los efectos nocivos de estas situaciones estresantes (LEIBOVICH DE FIGUEROA; SCHMIDT; MARRO, 2002; RICHAUD DE MINZI, 2004). 
Constanza Veloso-Besio; Washington Caqueo-Arancibia; Alejandra Caqueo-Urizar;

Zunilda Muñoz-Sánchez; Francisca Villegas-Abarzúa

\section{CONTEXTO EDUCATIVO}

En el campo educacional se presta cada vez mayor atención a la evaluación de las estrategias de afrontamiento, debido al reconocimiento de la importancia que adquiere el fomento de éstas en los jóvenes, sobre todo si se contemplan las presiones y el estrés creciente al que están sometidos en ambientes de estudio y trabajo cada vez más competitivos. En este marco, se han realizado investigaciones que intentan dar cuenta de la conducta de afrontamiento de los adolescentes (GÓMEZ et al., 2006; GONZÁLEZ et al., 2002; PHELPS; JARVIS, 1994; SOLIS; VIDAL, 2006) asumiendo que esto tiene importancia para toda la sociedad (MASSONE; GONZÁLEZ, 2003).

Asimismo, se viene reconociendo que la utilización de estas estrategias no es ajena a lo que acontece en el ámbito escolar ya que se relaciona con cuestiones tales como la obtención, el análisis y la organización de la información; la comunicación de ideas e informaciones; la planificación, el trabajo en equipo y la resolución de problemas o cómo buscar el consejo de los demás o hablar de los propios problemas con otros (MASSONE; GONZÁLEZ, 2003).

El contexto educativo en que se desenvuelve el adolescente pudiera tener un impacto importante en la elección de las estrategias de afrontamiento utilizadas por los jóvenes (LEIBOVICH DE FIGUEROA; SHUFER; MUIÑOZ, 1998), ya daban cuenta de algo parecido al establecer que el contexto es un factor interviniente y determinante del comportamiento, por lo que resultaría conveniente tomar en cuenta los ambientes percibidos por el sujeto. Asimismo, en una revisión realizada por Compas et al. (2001) se plantea la necesidad de enfocarse en los contextos sociales en los cuales los sujetos deben afrontar situaciones estresantes.

Es así que para la presente investigación se consideró como contexto educativo el tipo de Establecimiento Educacional en sus tres variantes, Privado, Particular Subvencionado y Municipal.

El sistema educativo chileno experimentó una profunda reforma en los albores de los '80s, al descentralizar la administración de los Establecimientos Escolares del sector público y transferirla a las Municipalidades. La reforma, su vez, permitió la incorporación del sector privado como oferente de educación, a través de un mecanismo de subvención por alumno. Dicha subvención financia a los establecimientos Particulares Subvencionados y Municipalizados. El sistema de subvenciones por estudiante tiene como objetivo cubrir el conjunto de gastos de operación de las escuelas, y a la vez promover la competencia entre los establecimientos por atraer y retener estudiantes, lo que debiera redundar en una mayor eficiencia y calidad de los servicios educativos (MIZALA; ROMAGUERA, 1998).

Esta política llevó a la creación de un gran número de Establecimientos Educacionales, generándose tres tipos de Establecimientos Educacionales: los Privados que se financian sólo en base a las contribuciones de los padres, son operados por el sector privado. A este tipo de Establecimiento asisten, en la ciudad de Arica, jóvenes de nivel socio económico medio alto; los Particulares Subvencionados que se financian con la subvención por alumno que otorga el Estado, son operados 
por el sector privado. Los adolescentes que asisten a este tipo de Establecimiento, en la ciudad de Arica, corresponden a un nivel socioeconómico medio bajo; y finalmente los Municipalizados que se financian con la subvención por alumno que otorga el Estado y son operados por los Municipios, asisten regularmente jóvenes de nivel socioeconómico bajo (MIZALA; ROMAGUERA, 1998).

Desde esta perspectiva, se vuelve necesario conocer cómo el contexto, en este caso los tipos de Establecimientos Educativos, puede influir en la utilización de estrategias de afrontamiento en los adolescentes. Si bien los trabajos disponibles (CASULLO; FERNÁNDEZ LIPOARCE, 2001; FIGUEROA et al., 2005; MASSONE; GONZÁLEZ, 2003) se enfocan en el estudio de las estrategias de afrontamiento de los adolescentes escolarizados, no se hallan investigaciones que analicen posibles efectos diferenciales en la elección de dichas estrategias entre tipos de Establecimientos. Es así que el presente estudio tiene como objetivo describir y analizar las estrategias de afrontamiento que presentan los adolescentes en los distintos Establecimientos Educacionales (Privado, Particular Subvencionado por el Estado y Municipal).

\section{MÉTodo}

\section{Participantes}

La muestra estuvo conformada por 323 adolecentes, estudiantes (184 hombres y 139 mujeres) de Octavo Básico a Cuarto Año Medio pertenecientes a Establecimientos Educacionales de la ciudad de Arica. De la muestra total, 90 participantes ( 58 hombres y 32 mujeres) correspondían a Establecimiento Privado; 114 participantes (56 hombres y 58 mujeres) pertenecían a Establecimiento Particular Subvencionado y 119 (70 hombres y 49 mujeres) correspondían a Establecimiento Municipal. La edad de los participantes se encontraba entre 12 y 18 años. La técnica de muestreo empleada fue no probabilística por disponibilidad.

\section{Instrumentos}

Se suministró la Escala de Afrontamiento para Adolescentes (ASC) (FRYDENBERG; LEWIS, 1997) en su forma general. Consta de 79 elementos de tipo cerrado, con una escala Likert de cinco opciones, de la cual se pueden extraer 18 estrategias. La consistencia interna es de 0.75 .

\section{Procedimiento}

El diseño corresponde a un estudio ex post facto retrospectivo para evaluar las estrategias de afrontamiento utilizadas por los adolescentes.

Para la recolección de los datos, en primer lugar, se enviaron cartas petitorio solicitando la colaboración en cada uno de los tres tipos de Establecimientos Educativos. Los cursos considerados incluyen desde octavo básico (enseñanza primaria) a cuarto año medio (enseñanza secundaria), de manera de obtener una muestra heterogénea. La administración del instrumento se realizó en horario de clases, de manera grupal y se garantizó el anonimato. 
Constanza Veloso-Besio; Washington Caqueo-Arancibia; Alejandra Caqueo-Urizar;

Zunilda Muñoz-Sánchez; Francisca Villegas-Abarzúa

\section{Resultados}

Se analizaron las estrategias de afrontamiento utilizadas por los adolescentes de la muestra de manera general. Se observó que los adolescentes de 12 a 18 años presentaron las siguientes estrategias como las más representativas del grupo (se citan en orden decreciente de acuerdo a la frecuencia de uso): buscar diversión relajante, preocuparse, distracción física, esforzarse y tener éxito, fijarse en lo positivo (Tabla 1).

Tabla 1 Descripción general de las estrategias utilizadas por los adolescentes

\begin{tabular}{|l|c|c|c|}
\hline Estrategia & Media & $\begin{array}{c}\text { Desviación } \\
\text { Estándar }\end{array}$ & Rango \\
\hline Buscar diversión relajante & 75,8 & 17,7 & $21-105$ \\
\hline Preocuparse & 71,7 & 14,5 & $20-100$ \\
\hline Distracción física & 69,0 & 22,0 & $21-105$ \\
\hline Esforzarse y tener éxito & 68,9 & 15,2 & $20-100$ \\
\hline Buscar pertenencia & 68,5 & 15,4 & $20-100$ \\
\hline Fijarse en lo positivo & 68,0 & 17,3 & $20-100$ \\
\hline Concentrarse en resolver el problema & 66,7 & 16,1 & $24-100$ \\
\hline Invertir en amigos íntimos & 65,1 & 17,0 & $20-100$ \\
\hline Hacerse ilusiones & 63,9 & 15,1 & $20-100$ \\
\hline Buscar apoyo social & 60,7 & 17,8 & $20-100$ \\
\hline Reservarlo para sí & 57,9 & 16,3 & $20-100$ \\
\hline Auto inculparse & 56,4 & 16,9 & $20-100$ \\
\hline Buscar apoyo espiritual & 51,9 & 16,5 & $20-100$ \\
\hline Buscar apoyo profesional & 50,7 & 18,4 & $15-100$ \\
\hline Ignorar el problema & 49,8 & 17,1 & $20-100$ \\
\hline Falta de afrontamiento & 46,2 & 14,2 & $20-92$ \\
\hline Reducción de la tensión & 42,1 & 15,1 & $20-100$ \\
\hline Acción Social & 41,2 & 15,5 & $20-90$ \\
\hline
\end{tabular}

Con la finalidad de obtener información más específica, se analizaron las estrategias de afrontamiento según tipo de Establecimiento Educacional. En la Tabla 2, se presentan los resultados descriptivos de dichos análisis. Se puede observar que las cinco estrategias más utilizadas por los adolescentes, de acuerdo al tipo de Establecimiento Educacional al que asisten, son prácticamente las mismas: buscar diversión relajante, preocuparse, distracción física, esforzarse y tener éxito y fijarse en lo positivo. Además de esto, se puede apreciar una tendencia del Establecimiento Particular Subvencionado a una mayor frecuencia de utilización de 12 de las 18 estrategias evaluadas. No obstante, es necesario analizar si estas diferencias son significativas o no (Ver Tabla 3). 
Tabla 2 Descripción de las estrategias utilizadas por los adolescentes según Tipo de Establecimiento

\begin{tabular}{|c|c|c|c|c|c|c|c|c|c|}
\hline \multirow[t]{3}{*}{ ESTRATEGIA } & \multicolumn{9}{|c|}{ TIPO DE ESTABLECIMIENTO } \\
\hline & \multicolumn{3}{|c|}{$\begin{array}{l}\text { Municipal } \\
\mathrm{N}=119\end{array}$} & \multicolumn{3}{|c|}{$\begin{array}{l}\text { Particular } \\
\text { Subven cionado } \\
\mathrm{N}=114\end{array}$} & \multicolumn{3}{|c|}{$\begin{array}{l}\text { Privado } \\
\mathrm{N}=90\end{array}$} \\
\hline & M & DS & Rango & M & DS & Rango & M & DS & Rango \\
\hline Buscar diversión relajante & 70,5 & 17,8 & $21-105$ & 79,8 & 17,2 & $35-105$ & 77,7 & 16,8 & $35-105$ \\
\hline Preocuparse & 68,1 & 14,8 & $32-100$ & 75,2 & 23,8 & $32-100$ & 71,1 & 14,3 & $20-100$ \\
\hline Distracción física & 66,5 & 22,4 & $21-105$ & 72,8 & 22,5 & $21-105$ & 67,5 & 20,7 & $21-105$ \\
\hline Esforzarse y tener éxito & 64,3 & 15,6 & $20-100$ & 73,7 & 13,7 & $44-100$ & 68,8 & 15,2 & $20-100$ \\
\hline Buscar pertenencia & 65,8 & 14,9 & $20-100$ & 72,4 & 13,8 & $24-100$ & 67,1 & 17,1 & $24-100$ \\
\hline Fijarse en lo positivo & 65,6 & 16,8 & $20-95$ & 72,2 & 16,4 & $35-100$ & 66,0 & 18,1 & $20-100$ \\
\hline C. Resolver el problema & 63,5 & 16,4 & $24-100$ & 68,5 & 15,8 & $32-100$ & 68,7 & 15,4 & $24-100$ \\
\hline Invertir en amigos íntimos & 61,1 & 17,3 & $20-100$ & 68,1 & 15,0 & $24-96$ & 66,4 & 18,2 & $24-100$ \\
\hline Hacerse ilusiones & 62,9 & 14,6 & $28-100$ & 66,5 & 14,3 & $32-96$ & 62,1 & 16,3 & $20-96$ \\
\hline Buscar apoyo social & 56,4 & 17,6 & $20-96$ & 65,7 & 16,9 & $24-96$ & 60,0 & 17,7 & $24-100$ \\
\hline Reservarlo para sí & 58,4 & 15,6 & $20-100$ & 58,1 & 16,0 & $20-100$ & 57,2 & 17,6 & $20-95$ \\
\hline Auto inculparse & 56,3 & 16,8 & $20-100$ & 56,5 & 17,4 & $25-100$ & 56,4 & 16,5 & $20-95$ \\
\hline Buscar apoyo espiritual & 54,5 & 16,6 & $20-100$ & 54,1 & 15,9 & $20-90$ & 45,7 & 15,8 & $20-95$ \\
\hline Buscar apoyo profesional & 48,5 & 18,9 & $20-90$ & 53,9 & 18,3 & $20-95$ & 49,7 & 17,5 & $15-100$ \\
\hline Ignorar el problema & 51,2 & 15,8 & $20-100$ & 50,2 & 18,7 & $20-100$ & 46,8 & 16,4 & $20-95$ \\
\hline Falta de afrontamiento & 47,3 & 14,4 & $20-92$ & 46,6 & 15,5 & $20-84$ & 43,3 & 12,7 & $20-80$ \\
\hline Reducción de la tensión & 43,2 & 15,6 & $20-100$ & 42,3 & 15,9 & $20-88$ & 40,3 & 13,3 & $20-72$ \\
\hline Acción Social & 40,9 & 15,9 & $20-80$ & 44,0 & 17,2 & $20-90$ & 37,9 & 12,0 & $20-75$ \\
\hline
\end{tabular}

Nota: $\mathrm{M}=$ media; $\mathrm{DS}=$ desviación estándar.

Al analizar diferencias en Estrategias de Afrontamiento por tipo de Establecimiento, se encontró que diez de ellas presentaron diferencias significativas según el tipo de Establecimiento. Más específicamente, ocho mostraron diferencias entre el Establecimiento Particular Subvencionado y el Municipal. Cinco entre el Particular Subvencionado y el Privado y dos indicaron diferencias significativas entre el Establecimiento Municipal y el Privado. 
Constanza Veloso-Besio; Washington Caqueo-Arancibia; Alejandra Caqueo-Urizar;

Zunilda Muñoz-Sánchez; Francisca Villegas-Abarzúa

Tabla 3 Diferencias entre estrategias de afrontamiento según tipo de Establecimiento Educativo

\begin{tabular}{|c|c|c|c|c|}
\hline $\begin{array}{ll}\text { Estrategias } & \text { de } \\
\text { Afrontamiento } & \end{array}$ & \multicolumn{2}{|l|}{ Tipo de Establecimiento } & $\begin{array}{l}\text { Diferencia } \\
\text { de medias }\end{array}$ & $\begin{array}{l}\text { Nivel de } \\
\text { significan cia } \\
(<0.05)\end{array}$ \\
\hline Buscar apoyo social & $\begin{array}{l}\text { Particular Subvencionado } \\
(65,7)\end{array}$ & $\begin{array}{l}\text { Municipal } \\
(56,4)\end{array}$ & $9,212 *$ & $0,000 *$ \\
\hline \multirow{2}{*}{$\begin{array}{l}\text { Esforzarse y tener } \\
\text { éxito }\end{array}$} & \multirow[t]{2}{*}{$\begin{array}{l}\text { Particular Subvencionado } \\
(73,7)\end{array}$} & $\begin{array}{l}\text { Municipal } \\
(64,3)\end{array}$ & $9,382 *$ & $0,000^{*}$ \\
\hline & & $\begin{array}{l}\text { Privado } \\
(68,8)\end{array}$ & $4,929^{*}$ & $0,046^{*}$ \\
\hline Preocuparse & $\begin{array}{l}\text { Particular Subvencionado } \\
(75,2)\end{array}$ & $\begin{array}{l}\text { Municipal } \\
(68,1)\end{array}$ & $6,421 *$ & $0,002 *$ \\
\hline $\begin{array}{l}\text { Invertir en amigos } \\
\text { íntimos }\end{array}$ & $\begin{array}{l}\text { Particular Subvencionado } \\
(61,1)\end{array}$ & $\begin{array}{l}\text { Municipal } \\
(68,1)\end{array}$ & $6,963^{*}$ & $0,005^{*}$ \\
\hline \multirow[t]{2}{*}{ Buscar pertenencia } & \multirow[t]{2}{*}{$\begin{array}{l}\text { Particular Subvencionado } \\
(72,4)\end{array}$} & $\begin{array}{l}\text { Municipal } \\
(67,1)\end{array}$ & $6,519 *$ & $0,003^{*}$ \\
\hline & & $\begin{array}{l}\text { Privado } \\
(67,1)\end{array}$ & $5,240^{*}$ & $0,040^{*}$ \\
\hline \multirow[t]{2}{*}{$\begin{array}{l}\text { Buscar } \\
\text { espiritual }\end{array}$} & $\begin{array}{l}\text { Particular Subvencionado } \\
(54,1)\end{array}$ & $\begin{array}{l}\text { Privado } \\
(45,7)\end{array}$ & $8,434 *$ & $0,001 *$ \\
\hline & $\begin{array}{l}\text { Municipal } \\
(54,5)\end{array}$ & $\begin{array}{l}\text { Privado } \\
(45,7)\end{array}$ & $8,765^{*}$ & $0,000^{*}$ \\
\hline \multirow[t]{2}{*}{ Fijarse en lo positivo } & \multirow[t]{2}{*}{$\begin{array}{l}\text { Particular Subvencionado } \\
(72,2)\end{array}$} & $\begin{array}{l}\text { Municipal } \\
(65,6)\end{array}$ & $6,647 *$ & $0,009^{*}$ \\
\hline & & $\begin{array}{l}\text { Privado } \\
(66,0)\end{array}$ & $6,249^{*}$ & $0,026^{*}$ \\
\hline \multirow[t]{2}{*}{$\begin{array}{ll}\text { Buscar } & \text { diversiones } \\
\text { relajantes } & \end{array}$} & $\begin{array}{l}\text { Particular Subvencionado } \\
(79,8)\end{array}$ & $\begin{array}{l}\text { Municipal } \\
(70,5)\end{array}$ & $9,354 *$ & $0,000^{*}$ \\
\hline & $\begin{array}{l}\text { Municipal } \\
(70,5)\end{array}$ & $\begin{array}{l}\text { Privado } \\
(77,7)\end{array}$ & $7,252 *$ & $0.008^{*}$ \\
\hline $\begin{array}{l}\text { Concentrarse en } \\
\text { resolver el problema }\end{array}$ & $\begin{array}{l}\text { Particular Subvencionado } \\
(68,5)\end{array}$ & $\begin{array}{l}\text { Municipal } \\
(63,5)\end{array}$ & $4,927 *$ & $0,049^{*}$ \\
\hline
\end{tabular}

Nota: Los cifras al interior de los paréntesis corresponden a las medias obtenidas en las estrategias de afrontamiento de acuerdo al tipo de Establecimiento Educacional.

\section{Discusión}

El objetivo de la investigación fue describir y analizar las estrategias de afrontamiento que presentan los adolescentes en los distintos establecimientos educacionales (Privado, Particular Subvencionado y Municipal). A partir de los resultados obtenidos se observa que los adolescentes estudiados enfrentan sus dificultades recurriendo a diversiones relajantes como escuchar música o ver televisión, o a través de la distracción física como el deporte. Por otra parte, existe un porcentaje importante que tiende a preocuparse y a trabajar intensamente.

Investigaciones anteriores como la realizada por Castellá, Carlotto y Goncalves (2007), destacan la búsqueda de pertenencia como una estrategia predominante, sin embargo, en el presente estudio la estrategia más frecuente fue preocuparse, perteneciente al estilo de afrontamiento focalizado en la evitación, estilo no considerado en el estudio realizado por Castellá, Carlotto y Goncalves (2007). 
En relación a las diferencias entre estrategias de afrontamiento según tipo de Establecimiento Educativo, se encontró que el Establecimiento Particular Subvencionado presentó claras diferencias en relación al Privado y Municipal, donde los adolescentes pertenecientes al Establecimiento Particular Subvencionado disponen de una mayor gama de estrategias focalizadas en la reducción del problema y la focalizada en las emociones, en comparación a los adolescentes pertenecientes a los otros dos Establecimientos mencionados anteriormente, lo que podría estar relacionado con el contexto psicosocial en el que se encuentran inmersos los jóvenes (COHEN; FIGUEROA, 2004) o al nivel socio económico al que pertenecen (RICHAUD DE MINZI, 2005). De acuerdo con los resultados de estas investigaciones, es posible plantear que los adolescentes de Establecimientos Particulares Subvencionados tienen padres con jornadas laborales intensas, por lo que no cuentan con ellos como modelos que exhiban las estrategias de afrontamiento adecuadas para hacer frente a situaciones estresantes, es así que la elección de las estrategias posiblemente responda a reiterados procesos de ensayo y error, probando durante este proceso distintas alternativas, hasta dar con aquella que resuelva, ya sea a corto o a largo plazo, la percepción de malestar. En cambio para los adolescentes de colegios Municipalizados, de condición socio económica baja, suelen estar insertos en un núcleo familiar en que los jefes de familia se ven afectados por serios problemas de desempleo, no llegando a constituirse en familias estables, o bien, abandonan el grupo familiar, quedando éste a cargo de mujeres. En este tipo de situación no existe o es muy limitada la socialización primaria que ofrece la familia (FIGUEROA et al., 2005), sumado a esto se tiene que, muchas veces estos jóvenes deben asumir roles adultos para los cuales no tienen la madurez suficiente ni están preparado psicológicamente (PICCO; GALENDE, 2001).

Por otra parte, los jóvenes de los Establecimientos Privado, cuentan con un ambiente cultural enriquecido, padres que responden a sus necesidades, a la vez, que se constituyen en modelos a seguir, presentándoles las estrategias adecuadas. De acuerdo con esto, es posible que estos jóvenes no tengan la necesidad de probar con distintas alternativas, como los de colegios Municipalizados, lo que redunde en una menor variedad de estrategias utilizadas. En relación a las estrategias focalizadas en la evitación se encontró homogeneidad en el uso que le dan los adolescentes a ésta, lo que podría ser explicado por la etapa vital que están pasando, donde debido a la gran cantidad de cambios y elecciones a los que se ven enfrentados, se les dificultan controlar sus emociones, acrecentándose la percepción de soledad y de no contar con el apoyo de los demás. Lo cual, les puede conducir a la elección de la evitación como la estrategia más efectiva.

Finalmente, este estudio logra relacionar aspectos importantes del contexto y su posible influencia en la elección de estrategias de afrontamiento por parte de los adolescentes.

Líneas futuras de investigación podrían apuntar al diseño de programas de intervención que consideren estas diferencias en las formas de afrontamiento con el objeto de favorecer mecanismos de resolución más efectivos, lo que podría incluso prevenir la aparición de posibles trastornos psicológicos en los adolescentes. 
Constanza Veloso-Besio; Washington Caqueo-Arancibia; Alejandra Caqueo-Urizar;

Zunilda Muñoz-Sánchez; Francisca Villegas-Abarzúa

Dentro de las limitaciones de este estudio, se plantea la dificultad en el acceso en la recolección de los datos, debido a la escasa cantidad - solo tres - de Establecimientos Educativos Privados, existentes en la ciudad. Además, se puede añadir que el Establecimiento Privado considerado en esta investigación, valora la autorregulación del alumnado en las áreas educativas y conductuales a diferencia de otros Establecimientos Privados de la ciudad de Arica que cuentan con una educación más estructurada y normativa.

Esta investigación ha sido financiada por el Proyecto de Iniciación en Investigación de la Universidad de Tarapacá N 571009.

\section{REFERENCIAS}

AMIRKAHN, J.; AUYEUNG, B. Coping with stress across the lifespan: absolute v/s relative changes in strategy. Journal of Applied Developmental Psychology, [S.1.], v. 28, n. 4, p. 298-317, 2007.

BALCÁZAR, P. et al. Percepción de los jóvenes para afrontar su vida. Interpsiquis, [S.1.], v. 20, n. 2, p. 30-39, 2006.

CASTEllá, J.; CARlotTO, M.; GONCALVES, S. Predictores de conductas sexuales de riesgo entre adolescentes. Interamericana Journal of Psychology, [S.1.], v. 41, n. 2, p. 161-66, 2007.

CASULLO, M.; FERNANDEZ LIPOARCE, M. Estrategias de afrontamiento en estudiantes adolescentes. Revista del Instituto de Investigaciones, [S.1.], v. 6, n. 1, p. 25-49, 2001.

CHICO, E. L. Optimismo disposicional como predictor de estrategias de afrontamiento. Psicothema, [S.1.], v. 14, n. 3, p. 544-550, 2002.

COHEN, S.; FIGUEROA, M. I. Estrategias y estilos de afrontamiento del estrés en adolescentes. Anales de Psicología, [S.1.], v. 20, n. 1, p. 29-52, 2004.

COMPAS, B. et al. Coping with stress during childhood and adolescence: problems, progress, and potential in theory and research. Psychollogical Bulletin, [S.1.], v. 127, n. 1, p. 87-127, 2001.

CORRECHÉ, M. S.; FANTIN, M.; FLORENTINO, M. T. Estilos de personalidad y estrategias de afrontamiento en adolescentes de una escuela privada de San Luis. Fundamentos en Humanidades, [S.1.], v. 6, n. 1, p. 159-176, 2005.

CUADRA PERALTA, A. et al. Teste de Rorschach: respostas diferenciales entre pacientes con y sin intento suicida. Fractal: Revista de Psicologia, Niterói, v. 21, n. 3, p. 475-486, 2009. Disponible en: <http://www.uff.br/periodicoshumanas/ index.php/Fractal/article/view/203/361>. Acesso em: 10 enero 2010. 
FEAGANS, L.; HUSSONG, A.; KEELEY, M. The adolescent coping process interview: measuring temporal and affective components of adolescent responses to peer stress. Journal of Adolescence, [S.1.], v. 31, n. 5, p. 641-657, 2008.

FIGUEROA, M. I. et al. Las estrategias de afrontamiento y su relación con el nivel de bienestar psicológico: un estudio con adolescentes de nivel socioeconómico bajo de Tucumán (Argentina). Anales de Psicología, Murcia, v. 21, n. 1, p. 66-72, 2005.

FORNS, M.; ZANINI, D. Coping y psicopatología: comparación entre adolescentes de la muestra general y sub-clínica. Terapia Psicológica, v. 22, n. 1, p. 1-10, 2004.

FRYDENBERG, E.; LEWIS, R. ACS: Escalas deAfrontamiento para Adolescentes. Adaptación española de Jaime Perena y Nicolás Seisdedos. [ACS: Adolescent Coping Scale (Spanish versión of Jaime Perena and Nicolás Seisdedos)]. Madrid: TEA, 1997.

FRYDENBERG, E. et al. Prevention is better than cure: coping skills training for adolescents at school. Educational Psychology in Practice, [S.1.], v. 20, n. 2, p. 117-134, 2004.

GÓMEZ, J. A. et al. Estrategias de afrontamiento en el inicio de la adolescencia y su relación con el consumo de drogas y la conducta problemática. International Journal of Clinical and Health Psychology, [S.1.], v. 6, n. 3, p. 581-597, 2006.

GONZÁLEZ, R., et al. Relación entre estrategias de afrontamiento y bienestar psicológico en adolescentes. Psicothema, Asturias, v. 14, n. 2, p. 363-368, 2002.

HAMPEL, P. Brief report: coping among austrian children and adolescents. Journal of Adolescence, [S.1.], v. 30, n. 5, p. 885-890, 2007.

HAMPEL, P.; PETERMANN, F. Perceived stress, coping, and adjustment in adolescents. Journal of Adolescent Health, [S.1.], v. 38, n. 4, p. 409-415, 2006.

ISON, M. El Afrontamiento: estrategias para el manejo del estrés. Revista IDEA, San Luis, v. 16, n. 37, p. 1-9, 2003.

LAZARUS, R. S.; FOLKMAN, S. Estrés y procesos cognitivos. Barcelona: Martínez Roca, 1984.

LEIBOVICHDEFIGUEROA, N.; SCHMIDT, V.I.; MARRO, C. T.Afrontamiento. En: _. El "malestar" y su evaluación en diferentes contextos. Buenos Aires: Eudeba, 2002. p. 45-59.

LEIBOVICH DE FIGUEROA, N.; SHUFER, M.; MUIÑOZ, R. Ecoevaluación Psicológica: las figuras humanas en contextos familiar y escolar. Buenos Aires: Eudeba, 1998. 
Constanza Veloso-Besio; Washington Caqueo-Arancibia; Alejandra Caqueo-Urizar;

Zunilda Muñoz-Sánchez; Francisca Villegas-Abarzúa

MASSONE, A.; GONZÁLEZ, G. Estrategias de afrontamiento (coping) y su relación con el logro académico y lengua en adolescentes de noveno año de educación general básica. Revista Iberoamericana de Educación, [S.1.], p. 1-7. 2003. Disponible en: <http://www.rieoei.org/deloslectores/378Massone.PDF $\geq$. Acesso em: 12 nov. 2009.

MIZALA, A.; ROMAGUERA, P. Desempeño escolar y elección de colegios: la experiencia chilena. Serie Econômica, Santiago, n. 36, 1998. Centro de Economía Aplicada, Departamento de Ingeniería Industrial, Facultad de Ciencias Físicas y Matemáticas, Universidad de Chile. Disponible en: $<$ http://decon.edu. uy/network/M00/mizala.pdf $>$. Acesso em: 10 enero 2010.

PHELPS, S.; JARVIS, P. Coping in adolescence: empirical evidence for a theoretically based approach to assessing coping. Journal of Youth and Adolescence, [S.1.], v. 23 n. 3, p. 359-371, 1994.

PICCO, E.; GALENDE, B. Trabajo infantil y su impacto en la constitución subjetiva. Kairós, [S.1.], v. 5, n. 8, 2001.

PINHEIRO, F. A.; TROCCOLI, B. T.; TAMAYO, M. R. Coping measurement in occupational setting. Psicología: Teoría e Pesquisa, [S.1.], v. 19, n. 2, p. 153-158, 2003.

PRITCHARD, M.; WILSON, G. Do coping styles change during the first semester of college? Journal of Social Psychology, [S.1.], v. 146, n. 1, p. 125-127, 2006.

RICHAUD DE MINZI, M. C. Development of coping resources in childhood and adolescents. Interdisciplinaria, [S.1.], n. esp., p. 63-74, 2004.

RICHAUD DE MINZI, M. C. Estilos parentales y estrategias de afrontamiento en niños. Revista Latinoamericana de Psicología, [S.1.], v. 37, n. 1, p. 47-58, 2005.

SOLIS, C.; VIDAL, A. Estrategias de afrontamiento en adolescentes. Revista de Psiquiatría y Salud Mental Hermilio Valdizan, [S.1.], v. 7, n. 1, p. 33-39, 2006.

WILLIAMS, K \& McGILLICUDDY-De Lisi, A. Coping Strategies in Adolescents. Journal of Applied Developmental Psychology, [S.1.], v. 20, n. 4, p. 537-549, 2000 .

ZIMMER-GEMBECK, M.; LOCKE, E. The socialization of adolescent coping behaviors: relationship with families and teachers. Journal of Adolescence, [S.1.], v. 30, n. 1, p. 1-16. 2007.

Recebido em: fevereiro de 2010

Aceito em: abril de 2010 